\title{
Antitumor activity of a combination of trastuzumab (Herceptin) and oral fluoropyrimidine $S-1$ on human epidermal growth factor receptor 2-overexpressing pancreatic cancer
}

\author{
HIROYUKI SAEKI ${ }^{1}$, SHUNSUKE YANOMA ${ }^{2}$, SHOUJI TAKEMIYA ${ }^{3}$, YUKIO SUGIMASA ${ }^{3}$, \\ MAKOTO AKAIKE $^{3}$, NORIO YUKAWA ${ }^{1}$, YASUSHI RINO ${ }^{1}$ and TOSHIO IMADA ${ }^{1}$ \\ ${ }^{1}$ Department of General Surgery, Yokohama City University, 3-9 Hukuura, Kanazawa-ku, Yokohama, \\ Kanagawa 236-0004; ${ }^{2}$ Research Institute, ${ }^{3}$ Department of Gastrointestinal Surgery, \\ Kanagawa Cancer Center, 1-1-2 Nakao, Asahi-ku, Yokohama, Kanagawa 241-0815, Japan
}

Received February 7, 2007; Accepted March 19, 2007

\begin{abstract}
The cytotoxic effect of trastuzumab in combination with oral fluoropyrimidine S-1 on human epidermal growth factor receptor 2 (HER2)-overexpressing human pancreatic cancer cell line TRG in vitro and in vivo was investigated. HER2 expression in TRG was analyzed by RT-PCR and flow cytometry. For in vitro experiments, 5-fluorouracil (5-FU) was used instead of S-1. In vivo studies were conducted with TRG xenografts in athymic mice. Trastuzumab $(10 \mathrm{mg} / \mathrm{kg})$ was administered intraperitoneally once a week for 4 weeks. S-1 $(10 \mathrm{mg} / \mathrm{kg})$ was administered orally 5 days a week for 4 weeks. The results showed that TRG cells were positive for HER 2 mRNA and overexpressed HER2 protein. Either trastuzumab or 5-FU concentration-dependently inhibited the growth of TRG cells. The combination of trastuzumab and 5-FU resulted in a significant inhibition of growth of TRG cells compared to either agent alone $(\mathrm{P}<0.001)$. Incubation of TRG cells with peripheral blood mononuclear cells after treatment with trastuzumab enhanced the antiproliferative effect of trastuzumab, which could be the result of antibodydependent cellular cytotoxicity. The combination of trastuzumab and $\mathrm{S}-1$ resulted in a significant reduction in xenograft volume compared to each agent alone $(\mathrm{P}<0.0001)$. In conclusion, this study showed that combination therapy with trastuzumab and S-1 may be effective for HER2-overexpressing pancreatic cancer patients.
\end{abstract}

Correspondence to: Dr Hiroyuki Saeki, Department of General Surgery, Yokohama City University, 3-9 Hukuura, Kanazawa-ku, Yokohama, Kanagawa 236-0004, Japan

E-mail: saekihiroyuki@yahoo.co.jp

Key words: trastuzumab, S-1, human epidermal growth factor receptor 2, pancreatic cancer, chemotherapy

\section{Introduction}

HER2 (human epidermal growth factor receptor 2) is a transmembrane tyrosine kinase receptor, which regulates cell growth and differentiation. A ligand binds to HER2 receptor, and HER2 forms a heterodimer with other receptors of the HER family (HER1, HER3, HER4), causing it to mediate signaling to cancer cells to proliferate. HER2 is overexpressed in $25-30 \%$ of breast cancers and is associated with poor prognosis for breast cancer patients (1-3). The anti-HER2 antibody trastuzumab (Herceptin) inhibits tumor growth in HER2-overexpressing breast cancer (4), and the response rate of trastuzumab alone for HER2-overexpressing breast cancer patients is $15-26 \%(5,6)$. Since the mechanism of action of trastuzumab is different from that of traditional chemotherapeutic agents, trastuzumab is expected to enhance the antitumor effect of chemotherapeutic agents without increasing their toxicity. Therefore, various combinations of trastuzumab and chemotherapies have been tried for breast cancer patients $(7,8)$. In pancreatic cancer, HER2 is overexpressed in $17-45 \%$ of the patients (9-13). However, the efficacy of trastuzumab as a single agent or in combination with chemotherapy for pancreatic cancer patients has not been fully evaluated. In a phase II study of gemcitabine and trastuzumab for pancreatic cancer patients, the response rate of trastuzumab and gemcitabine was similar to gemcitabine alone (14).

S-1 is an oral preparation of 5-fluorouracil (5-FU) consisting of tegafur (a prodrug of 5-fluorouracil), 5-chloro2,4-dihydroxypyridine, which inhibits the degradation of 5-fluorouracil, and potassium oxonate, which reduces gastrointestinal toxicity. Hayashi et al (15) reported that the response rates of S-1 alone and the combination of S-1 and cisplatin for pancreatic cancer patients were 20.0 and $57.1 \%$, respectively.

In this study, we investigated the antitumor activity of trastuzumab and S-1 in HER2-overexpressing human pancreatic cancer in vitro and in vivo.

\section{Materials and methods}

Compounds. Trastuzumab (Genentech, Inc., San Francisco, CA) was provided by Chugai Pharmaceutical (Tokyo, Japan). 
S-1 was from Taiho Pharmaceutical Co., Ltd. (Tokyo, Japan) and 5-fluorouracil was from Kyowa Hakko Co. (Tokyo, Japan).

Cell line. The human pancreatic cancer cell line TRG was established from pancreatic ductal adenocarcinoma resected from a pancreatic cancer patient at Kanagawa Cancer Center. Cells were cultured at $37^{\circ} \mathrm{C}$ with $5 \% \mathrm{CO}_{2}$ in RPMI-1640 complete medium, consisting of RPMI-1640 (Life Technologies, Inc., Gaithersburg, MD) supplemented with $1 \%$ glutamine, 100 units/ ml penicillin, $100 \mu \mathrm{g} / \mathrm{ml}$ streptomycin, and $10 \%$ fetal bovine serum (Sigma Chemical Co., St. Louis, MO).

Peripheral blood mononuclear cells (PBMCs). PBMCs were isolated from the blood of a healthy volunteer on FicollHypaque density gradients using LSM (ICN/Cappel, Aurora, $\mathrm{OH})$. The cells were cultured at $37^{\circ} \mathrm{C}$ with $5 \% \mathrm{CO}_{2}$ in the RPMI-1640 complete medium described above.

Animals. Five-week-old male nude mice (BALB/c) were purchased from Charles River Japan, Inc. (Yokohama, Japan). These mice were bred and maintained in a 12-h darklight cycle animal facility with controlled temperature (22$26^{\circ} \mathrm{C}$ ) and humidity (40-70\%) at Kanagawa Cancer Center. All animal experiments were conducted in accordance with the guidelines of the ethics committee at the Kanagawa Cancer Center.

HER1 (EGFR), HER2, HER3, and HER4 RNA expression in TRG cells analyzed by RT-PCR. Total RNA was extracted from TRG cells using TRIzol reagent (Invitrogen Corp., Carlsbad, CA). cDNA was synthesized with oligodT primer and Superscript II reverse transcriptase (Invitrogen Corp.). PCR amplifications were carried out in a T-Personal thermal cycler (Biometra, Göttingen, Germany) with Plattinum Taq DNA polymerase (Invitrogen Corp.). The following primers were used: HER1, forward (5' CCT GAG CTC TCT GAG TGC AAC C 3') and reverse (5' AGA TAC TCG GGG TTG CCC ACT G 3'); HER2, forward (5' CGG GAG ATC CCT GAC CTG CTG GAA 3') and reverse (5' CTG CTG GGG TAC CAG ATA CTC CTC 3'); HER3, forward (5' TGG GGA GTC TTG CCA GGA GTC 3') and reverse (5' GAG GAG GGA GTA CCT TTG AG 3'); and HER4, forward (5' CCT CTC CTT CCT GCG GTC TGT 3') and reverse (5' AAG TCT GGC AAT GAT TTT CTG TGG G 3') (16). Thermal cycling conditions for HER1, HER2 and HER3 were as follows: denaturation at $94^{\circ} \mathrm{C}$ for $30 \mathrm{sec}$; annealing at $60^{\circ} \mathrm{C}$ for $30 \mathrm{sec}$; and extension at $72^{\circ} \mathrm{C}$ for $1 \mathrm{~min}$ for 28 cycles. For HER4, the same conditions were used except that the PCR annealing temperature was $55^{\circ} \mathrm{C}$. An additional PCR amplification directed at the glucose-6-phosphate dehydrogenase (G6PDH) gene was performed as a control using primers forward $\left(5^{\prime}\right.$ CTG ACC TAC GGC AAC AGA TA3') and reverse (5' AAC CCA CTC TCT TCA TCA GC') under the following conditions: denaturation at $94^{\circ} \mathrm{C}$ for $15 \mathrm{sec}$; annealing at $55^{\circ} \mathrm{C}$ for $15 \mathrm{sec}$; and extension at $72^{\circ} \mathrm{C}$ for $30 \mathrm{sec}$ for 30 cycles. PCR products were analyzed by electrophoresis on a $1.5 \%$ agarose gel stained with ethidium bromide and visualized under UV light. The expected amplicon sizes were 315, 300, 320, 404 and 238 bp for HER1, HER2, HER3, HER4 and G6PDH, respectively.
Expression level of HER2 protein in TRG cells and the effect of trastuzumab and 5-fluorouracil on the HER2 expression level analyzed by flow cytometry. HER 2 protein expression in TRG cells and the change in the HER 2 expression level following exposure to trastuzumab alone ( 1 and $10 \mu \mathrm{g} / \mathrm{ml}), 5-$ fluorouracil alone $(1$ and $10 \mathrm{ng} / \mathrm{ml})$ and the combination of trastuzumab and 5-fluorouracil were analyzed by flow cytometry. Cells $\left(1 \times 10^{6}\right)$ were suspended in $20 \mu \mathrm{l}$ of Hank's balanced solution (HBS) containing 10\% FBS. Anti-erbB2/ HER-2 mouse monoclonal antibody (Upstate Biotechnology, Inc., Lake Placid, NY) which was used as the primary antibody was added, and the cells were incubated for $30 \mathrm{~min}$ on ice. After washing three times with HBS containing $10 \%$ FBS, the cells were suspended in $20 \mu 1$ of HBS containing $10 \%$ FBS. Fluorescein isothiocyanate (FITC)-conjugated antimouse IgG monoclonal antibody (Becton Dickinson Labware, Lincoln Park, NJ) as a secondary antibody was added, and the cells were incubated for $30 \mathrm{~min}$. After washing with HBS containing $10 \%$ FBS twice, the cells were re-suspended in $1 \mathrm{ml}$ of HBS containing 10\% FBS. The expression level of HER2 was analyzed by flow cytometry using FACS (Becton Dickinson Labware). Unstained (autofluorescence control) cells and cells stained with the secondary antibody alone (secondary control) were analyzed by flow cytometry for the control.

Effect of trastuzumab and 5-fluorouracil on proliferation of human pancreatic cancer cells in vitro. TRG cells $\left(1 \times 10^{4}\right)$ were seeded in each well of a BD Falcon 96-well microplate (Becton Dickinson Labware). Cells were cultured in RPMI1640 supplemented with $10 \%$ fetal bovine serum. After a 1-day incubation at $37^{\circ} \mathrm{C}$ in a $5 \% \mathrm{CO}_{2}$ incubator, various concentrations of trastuzumab $(0,0.1,1,10$ and $100 \mu \mathrm{g} / \mathrm{ml})$ or 5fluorouracil $(0,1,10,100$ and $1000 \mathrm{ng} / \mathrm{ml})$ were added. After 3 days of incubation at $37^{\circ} \mathrm{C}$ in a $5 \% \mathrm{CO}_{2}$ incubator, the proliferation of the cells was measured by MTT [3-(4,5Dimethyl-2-thiazolyl)-2,5-diphenyl-2 $\mathrm{H}$-tetrazolium bromide] assay.

Effect of combinations of trastuzumab and 5-fluorouracil on human pancreatic cancer cells in vitro. After a 1-day incubation of $5 \times 10^{5}$ TRG cells seeded in a $10-\mathrm{cm}$ dish with $5 \mathrm{ml} 10 \%$ fetal bovine serum at $37^{\circ} \mathrm{C}$ in a $5 \% \mathrm{CO}_{2}$ incubator, trastuzumab and 5-fluorouracil were added as single agents and in combination. No agent was added to the control. After 3 days of incubation at $37^{\circ} \mathrm{C}$ in a $5 \% \mathrm{CO}_{2}$ incubator, cells were harvested by trypsinization and were counted. Experiments were performed in triplicate. Percentages of control cell proliferation (cell number/mean cell number in controls $\mathrm{x}$ 100) were compared in each group.

Antibody-dependent cellular cytotoxicity (ADCC) of trastuzumab on human pancreatic cancer cells in vitro. After a 1-day incubation of $1 \times 10^{6}$ TRG cells in $5 \mathrm{ml} 10 \%$ fetal bovine serum at $37^{\circ} \mathrm{C}$ in a $5 \% \mathrm{CO}_{2}$ incubator, trastuzumab and 5 -fluorouracil were added as single agents and in combination. No agent was added to the control. After 2 days of incubation at $37^{\circ} \mathrm{C}$ in a $5 \% \mathrm{CO}_{2}$ incubator, cells were washed to remove the added agent. In order to analyze antibody-dependent 
A

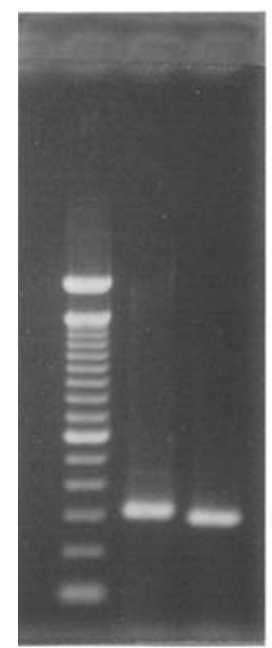

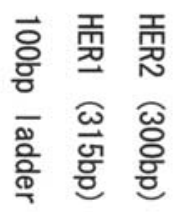

B

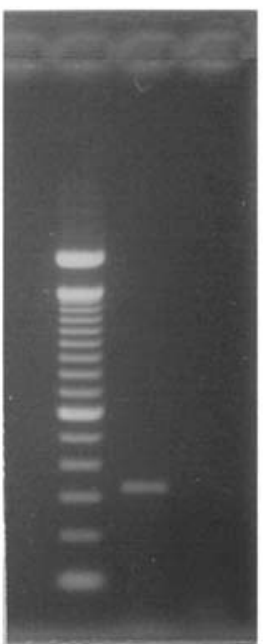

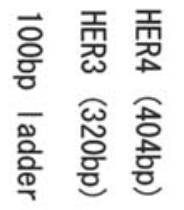

C

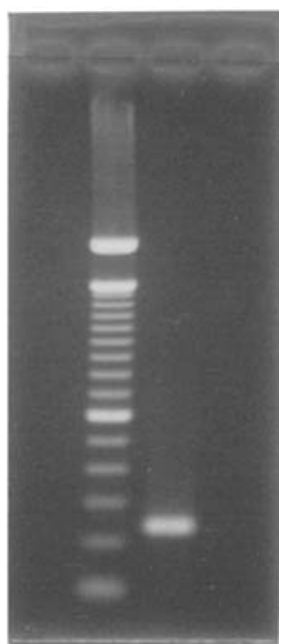

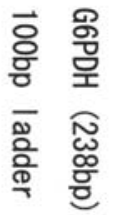

Figure 1. HER1, HER2, HER3, and HER4 mRNA expression in TRG cells was analyzed by RT-PCR. Total RNA was extracted from TRG cells and cDNA was synthesized. The cDNA was subjected to RT-PCR with primers specific for HER1, HER2, HER3, and HER4. PCR products were analyzed by electrophoresis on 1.5\% agarose gel stained with ethidium bromide and visualized under UV light. TRG cells were positive for HER1, HER2, and HER3, but not for HER4 (A, B). An additional PCR amplification directed at the glucose-6-phosphate dehydrogenase (G6PDH) gene was performed as a control (C).

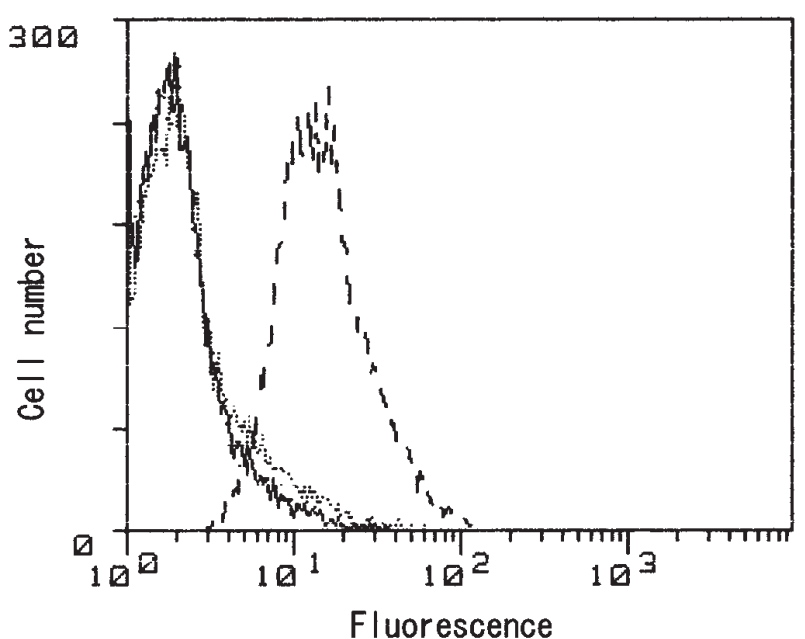

Figure 2. HER2 protein expression in TRG cells was analyzed by flow cytometry. Unstained (autofluorescence control -) cells stained with the secondary FITC-conjugated antibody alone (secondary control $\cdots \cdots \cdot$ ) and cells stained with anti-HER2 mouse monoclonal antibody, followed by the secondary antibody (-----) were analyzed by flow cytometry. TRG cells overexpressed HER2 protein.

cellular cytotoxicity (ADCC) with trastuzumab, $1 \times 10^{7}$ peripheral blood mononuclear cells (PBMCs) were added to each group. After $12 \mathrm{~h}$ of incubation at $37^{\circ} \mathrm{C}$ in a $5 \% \mathrm{CO}_{2}$ incubator, cells were counted. Experiments were performed in triplicate. Percentages of control cell proliferation were compared in each group.
Effect on human pancreatic cancer xenografts of a combination of trastuzumab and $S-1$. The in vivo effects of trastuzumab and S-1 as single agents and in combination were tested in a human pancreatic cancer xenograft model in athymic mice. The viable TRG cells $\left(5 \times 10^{6}\right)$ were injected subcutaneously into the mediodorsal regions of athymic mice. After a week of subcutaneous tumor growth, the mice were randomized into three treatment groups and a control group of 10 mice each, and the treatment was started. Trastuzumab was given intraperitoneally once a week for 4 weeks at a dose of $10 \mathrm{mg} / \mathrm{kg}$. S-1 was given orally 5 days a week for 4 weeks at a dose of $10 \mathrm{mg} / \mathrm{kg}$. The control group received no treatment. Tumor size and body weight were measured once a week, and tumor volume was calculated by the formula $1 / 2$ $\mathrm{x}$ large diameter $\mathrm{x}$ (small diameter $)^{2}$.

Statistical analysis. Results were expressed as the means \pm SE. Statistical significance was determined by the Student's ttest in comparing treatment groups. A P value $<0.05$ was accepted as statistically significant.

\section{Results}

HER1 (EGFR), HER2, HER3, and HER4 RNA expression in $T R G$ cells analyzed by RT-PCR. The mRNA expression of HER1, HER2, HER3, and HER4 was analyzed by RT-PCR. TRG cells were positive for HER1, HER2, and HER3, but not for HER4 (Fig. 1).

Expression level of HER2 protein in TRG cells and the effect of trastuzumab and 5-fluorouracil on the HER2 expression 
A
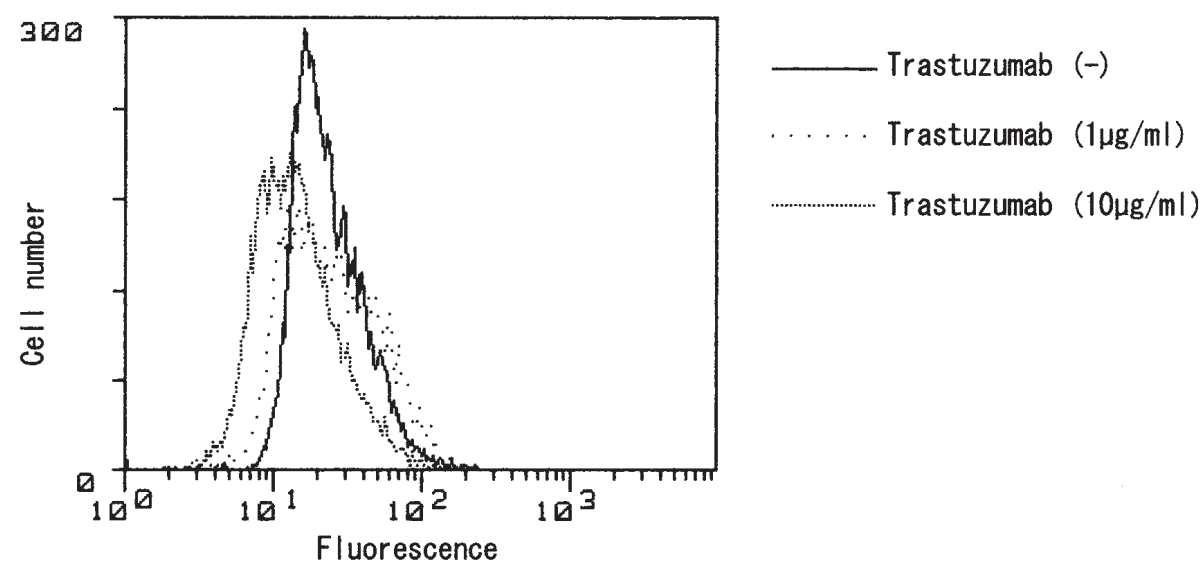

B
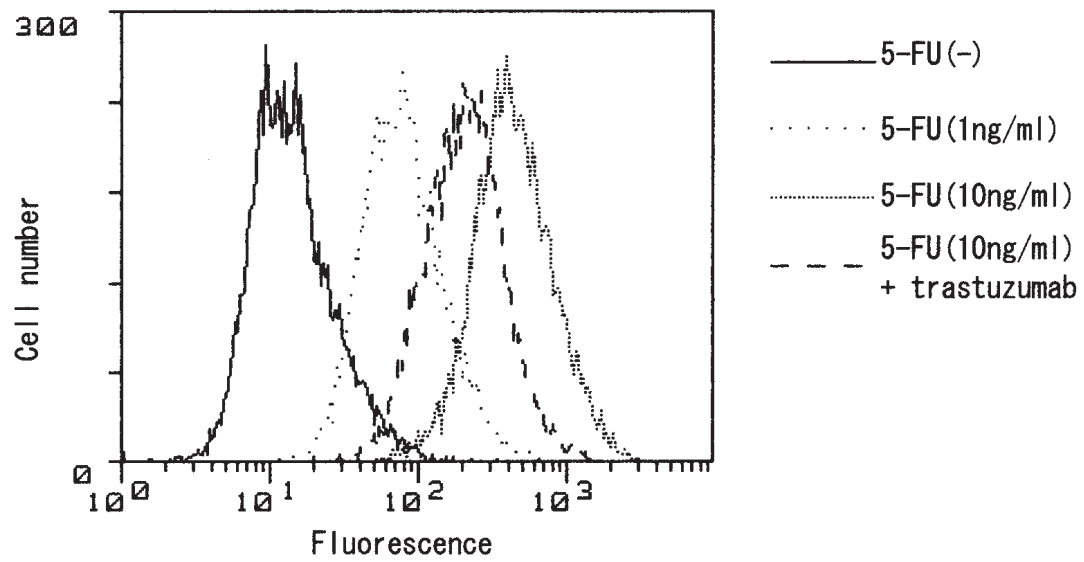

Figure 3 . The changes in the HER2 expression level following exposure to trastuzumab (1 and $10 \mu \mathrm{g} / \mathrm{ml})$ and/or 5-fluorouracil ( 1 and $10 \mathrm{ng} / \mathrm{ml})$ were analyzed by flow cytometry. Although the HER2 level of TRG cells did not change after treatment with trastuzumab alone (A), the HER2 level was increased after treatment with 5-fluorouracil alone and with a combination of trastuzumab and 5-fluorouracil (B).

level analyzed by flow cytometry. TRG cells overexpressed HER2 protein in flow cytometric analysis (Fig. 2). Although the HER2 level of TRG cells did not change after treatment with trastuzumab alone, the HER2 level was increased after treatment with 5-fluorouracil alone and with a combination of trastuzumab and 5-fluorouracil (Fig. 3).

Effect of trastuzumab and 5-fluorouracil on proliferation of human pancreatic cancer cells in vitro. TRG cells were cultured in the presence of $0.1,1,10$, and $100 \mu \mathrm{g} / \mathrm{ml}$ trastuzumab or 1, 10, 100, and $1000 \mathrm{ng} / \mathrm{ml} \mathrm{5-fluorouracil.}$ After 4 days of incubation, MTT assay was performed. Either trastuzumab or 5-fluorouracil concentration-dependently inhibited the growth of TRG cells (Fig. 4).

Effect of a combination of trastuzumab and 5-fluorouracil on human pancreatic cancer cells in vitro. TRG cells were cultured in the presence of $1 \mu \mathrm{g} / \mathrm{ml}$ trastuzumab and $10 \mathrm{ng} / \mathrm{ml}$ 5-fluorouracil alone and in combination, and the growth inhibition effects were compared. The combination of trastuzumab and 5-fluorouracil resulted in significant inhibition of the growth of TRG cells compared to either agent alone ( $\mathrm{P}=0.0004$ vs. 5-fluorouracil alone, $\mathrm{P}=0.0006$ vs. trastuzumab alone) (Fig. 5).
Antibody-dependent cellular cytotoxicity (ADCC) of trastuzumab on human pancreatic cancer cells in vitro. After incubation of TRG cells with trastuzumab and 5-fluorouracil, PBMCs were added to the TRG cells and the growth inhibition effects were compared. The inhibition effect of the trastuzumab group $(\mathrm{P}=0.001)$ and 5-fluorouracil group $(\mathrm{P}=0.009)$ in the presence of PBMSc was enhanced compared to that without PBMCs (Fig. 5). Although the inhibition effect in the trastuzumab group and the 5-fluorouracil group was not significantly different when PBMCs were not added, the inhibition effect in the trastuzumab group was greater than in the 5-fluorouracil group $(\mathrm{P}=0.06)$ in the presence of PBMCs. In the combination group, the enhancing effect on growth inhibition induced by PBMCs was not observed.

Effect of the combination of trastuzumab and $S-1$ on human pancreatic cancer xenografts. The in vivo effects of trastuzumab and S-1 alone and in combination were tested in a TRG subcutaneous xenograft model in athymic mice. Trastuzumab was given intraperitoneally once a week for 4 weeks at a dose of $10 \mathrm{mg} / \mathrm{kg}$. S-1 was given orally 5 times a week for 4 weeks at a dose of $10 \mathrm{mg} / \mathrm{kg}$. Two mice in the control group that received no treatment died within 4 weeks. The tumor volumes at 4 weeks in the three treatment groups 

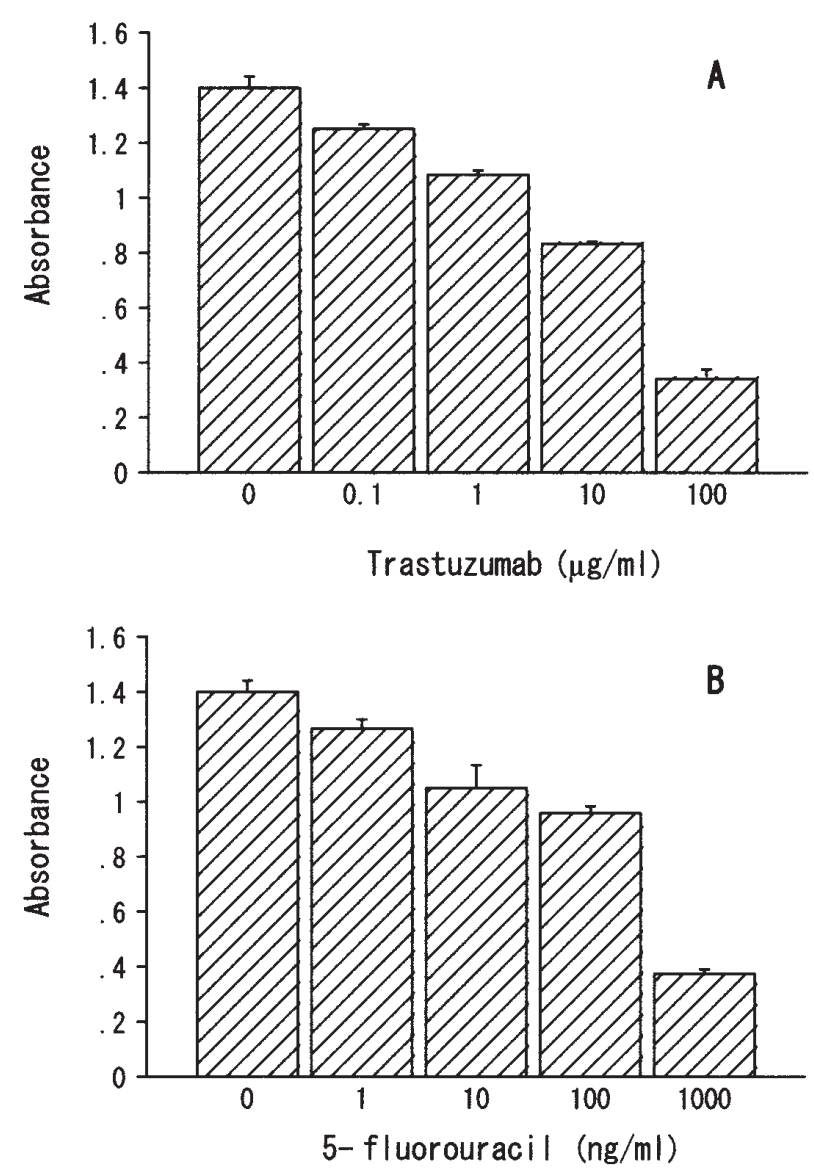

Figure 4. TRG cells were incubated with various concentrations of trastuzumab $(0.1,1,10,100 \mu \mathrm{g} / \mathrm{ml})$ or 5 -fluorouracil $(1,10,100,1000 \mathrm{ng} /$ $\mathrm{ml}$ ). The proliferation of cells was measured by MTT [3-(4,5-Dimethyl-2thiazolyl)-2,5-diphenyl- $2 \mathrm{H}$-tetrazolium bromide] assay. Either trastuzumab or 5-fluorouracil concentration-dependently inhibited the growth of TRG cells.

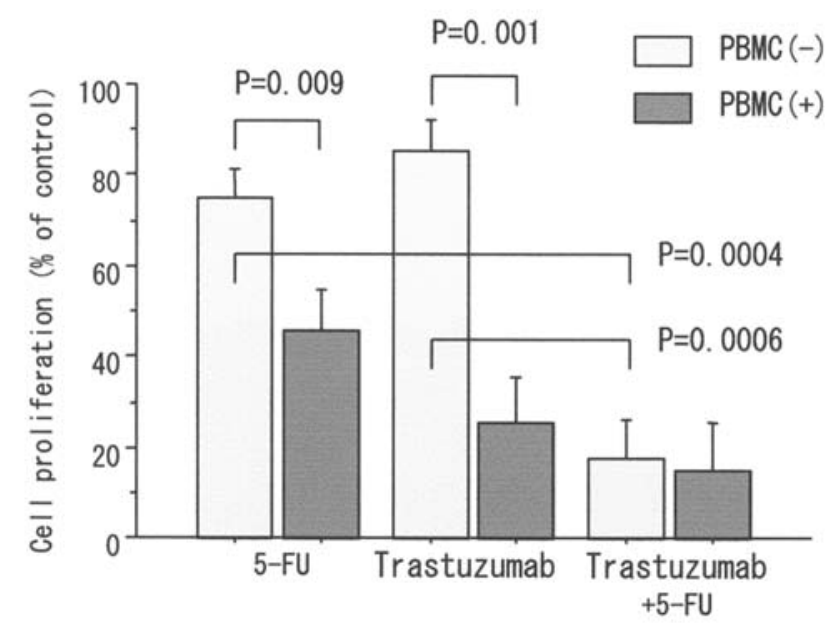

Figure 5. Effect of the combination of trastuzumab and 5-fluorouracil on TRG cells in vitro. TRG cells incubated with trastuzumab and/or 5fluorouracil and cell proliferation (percentages of control) were compared in each group. The combination of trastuzumab and 5-fluorouracil significantly inhibited cell proliferation of TRG compared to either agent alone $(\mathrm{P}=0.0006$ and $\mathrm{P}=0.0004$, respectively). TRG cells incubated with trastuzumab and/or 5-fluorouracil, followed by PBMCs and cell proliferation were compared in each group. The addition of PBMCs to TRG cells enhanced antiproliferative effects in 5-fluorouracil alone $(\mathrm{P}=0.009)$ and trastuzumab alone $(\mathrm{P}=0.001)$. were significantly reduced as compared with the control group $(\mathrm{P}<0.01)$ (Fig. 6). The combination of trastuzumab and S-1 resulted in a significant reduction $(\mathrm{P}<0.0001)$ in tumor volume compared to either agent alone. Weight loss in the combination group was significantly less than in the control group $(\mathrm{P}=0.0004)$ and the $\mathrm{S}-1$ group $(\mathrm{P}<0.0001)$, which demonstrated that the combination of trastuzumab and S-1 probably did not increase toxicity (Fig. 7).

\section{Discussion}

Although chemotherapy for pancreatic cancer patients has not been established, Hayashi et al (15) recently reported that the response rates of S-1 alone and the combination of S-1 and cisplatin for pancreatic cancer patients were 20.0 and $57.1 \%$, respectively. Therefore $\mathrm{S}-1$ is one of the hopeful drugs for pancreatic cancer patients. S-1 is an oral preparation of 5fluorouracil consisting of tegafur, 5-chloro-2,4-dihydroxypyridine and potassium oxonate. Tegafur is metabolized into 5-fluorouracil in the liver in vivo. 5-chloro-2,4-dihydroxypyridine competitively inhibits the 5-fluorouracil degradative enzyme dihydropyrimidine dehydrogenase (DPD), resulting in the retention of a prolonged concentration of 5-fluorouracil in the blood. For in vitro experiments, we used 5-fluorouracil, its metabolite, instead of tegafur itself. 5-fluorouracil concentration-dependently inhibited the proliferation of TRG cells in vitro. In in vivo experiments, S-1 inhibited the growth of TRG xenografts in athymic mice. There are two possible mechanisms of action for 5-fluorouracil; interference with RNA function and inhibition of DNA synthesis. After intracellular conversion to 5-fluorouridine triphosphate (FUTP), 5-fluorouracil is incorporated into RNA and interferes with RNA processing. 5-fluorouracil is also converted to 5-fluoro2'-deoxyuridine monophosphate (FdUMP), whereby a complex is formed with thymidylate synthase (TS), inhibiting the function of TS and impairing DNA synthesis. The high activity of tumoral TS and DPD (an enzyme of 5-fluorouracil catabolism) reduces the antitumor effect of 5-fluorouracil (17). However, the activity of TS and DPD was not evaluated in this study.

HER2 is overexpressed in $17-45 \%$ of pancreatic cancer patients (9-13). TRG cells expressed HER1, HER2, and HER3 mRNA in RT-PCR and overexpressed HER2 protein in flow cytometry. HER2 forms heterodimers with other members of the HER family to stimulate intercellular signaling pathways. In pancreatic cancer tissue, HER-2 coexpression with HER-3 and HER-4 is frequently seen (18). Treatment of TRG cells with trastuzumab resulted in concentration-dependent growth inhibition in vitro. An antitumor effect of trastuzumab for TRG xenografts was also observed. HER2 down-regulation is one possible mechanism of the antitumor effect of trastuzumab, which has not yet been completely elucidated $(19,20)$. The binding of trastuzumab to HER2 proteins decreases expression of cell surface HER2, thereby weakening growth signaling and inhibiting cell proliferation. In this study, we analyzed the change in HER2 expression level after treatment of TRG cells with trastuzumab, but HER2 down-regulation was not observed. Therefore the action of trastuzumab against TRG cells was likely due to the prevention of dimer formation, initiation of G1 arrest and 


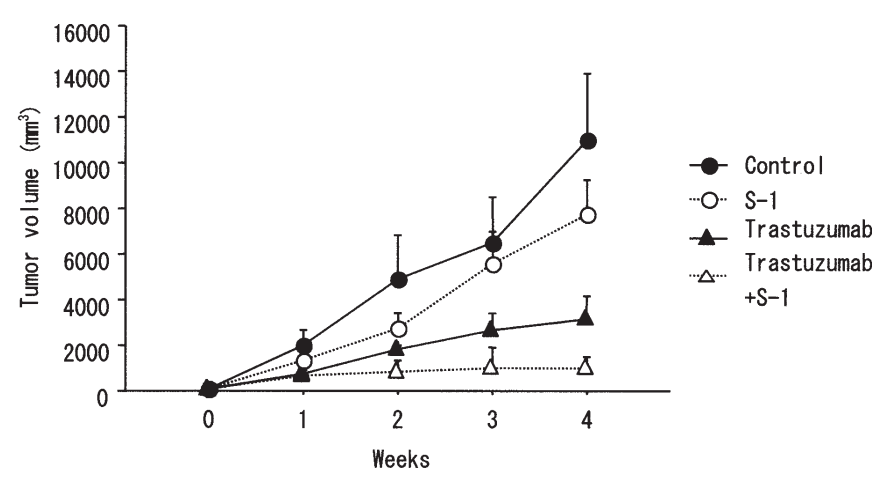

Figure 6. The in vivo effects of trastuzumab and S-1 as single agents and in combination were tested in a TRG subcutaneous xenograft model in athymic mice. Trastuzumab was given intraperitoneally once a week for 4 weeks at a dose of $10 \mathrm{mg} / \mathrm{kg}$. S-1 was given orally 5 times a week for 4 weeks at a dose of $10 \mathrm{mg} / \mathrm{kg}$. The tumor volumes at 4 weeks in the three treatment groups were significantly reduced as compared with the control group $(\mathrm{P}<0.01)$. The combination of trastuzumab and S-1 resulted in a significant reduction $(\mathrm{P}<0.0001)$ in tumor volume compared to either agent alone.

induction of p27, or prevention of HER2 cleavage (21), although further research is needed to explore these possibilities. Trastuzumab possesses the Fc domain of human IgG1, indicating that trastuzumab induces antibodydependent cellular cytotoxicity (ADCC), and inhibits cell growth (4), in addition to direct antitumor activity as previously described. In order to determine the ADCC induced by trastuzumab against TRG cells in vitro, we added peripheral blood mononuclear cells (PBMCs) to TRG cells after treatment with trastuzumab and 5-fluorouracil and analyzed cell growth inhibition. The growth inhibition in 5fluorouracil was increased by PBMCs as well as in trastuzumab. The enhanced growth inhibition in 5-fluorouracil, which is not an antibody-induced agent, seemed to be due to a direct cytotoxic effect of PBMCs. The enhanced effect by PBMCs in trastuzumab was greater than in 5-fluorouracil suggesting that ADCC could be operative in trastuzumab. In the combination group, the enhanced effect by PBMCs was not observed, since the cytotoxic effect of the combination of trastuzumab and 5-fluorouracil was too strong.

Previous studies have demonstrated synergistic or additive interactions between trastuzumab and a variety of chemotherapeutic drugs, such as paclitaxel, doxorubicin, and cisplatin (22-25), but few studies have reported the effect of trastuzumab with 5-fluorouracil. Pegram et al (25) reported that in an in vitro study using breast cancer cell line SK-BR-3, trastuzumab exhibited an antagonistic interaction against 5fluorouracil explained by the decreased effect with trastuzumab in the percentage of cells in the $S$ phase, where 5-fluorouracil exerts cytotoxic effects. Fujimoto-Ouchi et al (26) reported that the combination of trastuzumab and the oral fluoropyrimidine capecitabine demonstrated additive antiproliferative activity in human breast cancer KPL-4 and BT-474 xenograft models, even though in vitro treatment with trastuzumab and 5-fluorouracil showed antagonistic antiproliferative activity in these cell lines. They suggested that this discrepancy between the in vivo and in vitro experiments could be due to ADCC induced by trastuzumab. In the present in vitro experiment, the enhanced effect of the combination

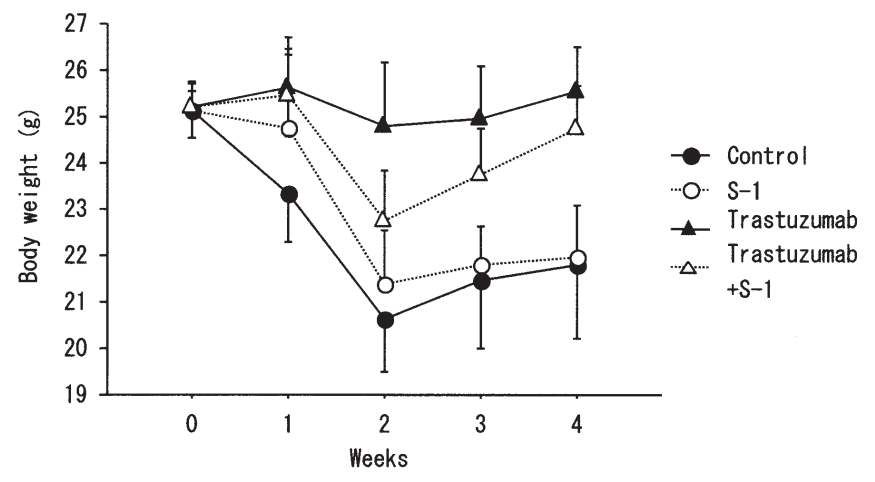

Figure 7. The body weight changes in mice treated with trastuzumab and S-1 as single agents and in combination. Body weight loss in the combination group was significantly less than in the control group $(\mathrm{P}=0.0004)$ and the $\mathrm{S}-1$ group $(\mathrm{P}<0.0001)$ demonstrating that the combination of trastuzumab and $\mathrm{S}-1$ probably did not increase toxicity.

of trastuzumab and 5-fluorouracil in the TRG cells was observed even without PBMCs, demonstrating that ADCC may not participate in the enhanced effect. Furthermore, the combination of S-1 and trastuzumab in in vivo experiments with TRG cells resulted in additive interactions.

One mechanism which may explain this enhanced effect of the combination of trastuzumab and 5-fluorouracil is upregulation induced by chemotherapeutic agents. Previous research has demonstrated that exposure of several cancer cell lines to doxorubicin resulted in an increase in expression of the EGFR (27). This phenomenon has been proposed to explain the synergistic cytotoxic effects of doxorubicin used in combination with anti-EGFR monoclonal antibodies. On the other hand, Pegram et al (25) reported that following exposure to doxorubicin, HER2 expression levels in SK-BR-3 breast carcinoma cells were unaltered. Treatment of TRG cells with 5-fluorouracil alone and the combination of 5-fluorouracil and trastuzumab resulted in an increase in HER2 expression. These data suggest that the enhanced effect with the combination of trastuzumab and 5-fluorouracil could be due to increased sensitivity for trastuzumab in TRG cells resulting from HER2 up-regulation. However, the interaction with trastuzumab and chemotherapeutic agents remains to be elucidated, and further research is needed to explore these effects.

In conclusion, we demonstrated the additive interaction with the combination of trastuzumab and 5-fluorouracil in vitro and the additive interaction with the combination of trastuzumab and S-1 in vivo using a HER2-overexpressing human pancreatic cell line. These data indicate that the clinical efficacy of the combination of trastuzumab and S-1 against HER2-overexpressing pancreatic cancer is worth investigating.

\section{References}

1. Slamon DJ, Clark GM, Wong SG, Levin WJ, Ullrich A and McGuire WL: Human breast cancer: correlation of relapse and survival with amplification of the HER-2/neu oncogene. Science 235: 177-182, 1987.

2. Slamon DJ, Godolphin W, Jones LA, et al: Studies of the HER$2 /$ neu proto-oncogene in human breast and ovarian cancer. Science 244: 707-712, 1989.

3. Seshadri R, Matthews C, Dobrovic A and Horsfall DJ: The significance of oncogene amplification in primary breast cancer. Int J Cancer 43: 270-272, 1989. 
4. Carter P, Presta L, Gorman CM, et al: Humanization of an antip185HER2 antibody for human cancer therapy. Proc Natl Acad Sci USA 89: 4285-4289, 1992.

5. Cobleigh MA, Vogel CL, Tripathy D, et al: Multinational study of the efficacy and safety of humanized anti-HER2 monoclonal antibody in women who have HER2-overexpressing metastatic breast cancer that has progressed after chemotherapy for metastatic disease. J Clin Oncol 17: 2639-2648, 1999.

6. Vogel CL, Cobleigh MA, Tripathy D, et al: Efficacy and safety of trastuzumab as a single agent in first-line treatment of HER2overexpressing metastatic breast cancer. J Clin Oncol 20: 719-726, 2002.

7. Burstein HJ, Kuter I, Campos SM, et al: Clinical activity of trastuzumab and vinorelbine in women with HER2-overexpressing metastatic breast cancer. J Clin Oncol 19: 2722-2730, 2001.

8. Slamon DJ, Leyland-Jones B, Shak S, et al: Use of chemotherapy plus a monoclonal antibody against HER2 for metastatic breast cancer that overexpresses HER2. N Eng1 J Med 344: 783-792, 2001.

9. Yamanaka Y, Friess H, Kobrin MS, Buchler M, Kunz J, Beger HG and Korc M: Overexpression of HER2/neu oncogene in human pancreatic carcinoma. Hum Pathol 24: 1127-1134, 1993.

10. Okada N, Ohshio G, Yamaki K, Imamura T and Imamura M: Elevated serum c-erbB-2 protein levels in patients with pancreatic cancer: correlation to metastasis and shorter survival. Oncology 52: 392-396, 1995.

11. Novotny J, Petruzelka L, Vedralova J, Kleibl Z, Matous B and Juda L: Prognostic significance of c-erbB-2 gene expression in pancreatic cancer patients. Neoplasma 48: 188-191, 2001.

12. Safran H, Steinhoff M, Mangray S, et al: Overexpression of the HER-2/neu oncogene in pancreatic adenocarcinoma. Am J Clin Oncol 24: 496-499, 2001.

13. Saxby AJ, Nielsen A, Scarlett CJ, Clarkson A, Morey A, Gill A and Smith RC: Assessment of HER-2 status in pancreatic adenocarcinoma: correlation of immunohistochemistry, quantitative real-time RT-PCR, and FISH with aneuploidy and survival. Am J Surg Pathol 29: 1125-1134, 2005.

14. Safran H, Iannitti D, Ramanathan R, et al: Herceptin and gemcitabine for metastatic pancreatic cancers that overexpress HER-2/neu. Cancer Invest 22: 706-712, 2004.

15. Hayashi K, Imaizumi T, Kuramochi H and Takasaki K: High response rates in patients with pancreatic cancer using the novel oral fluoropyrimidine S-1. Oncol Rep 9: 1355-1361, 2002.

16. Krahn G, Leiter U, Kaskel P, Udart M, Utikal J, Bezold G and Peter RU: Coexpression patterns of EGFR, HER2, HER3 and HER4 in non-melanoma skin cancer. Eur J Cancer 37: 251-259, 2001.
17. Beck A, Etienne MC, Cheradame S, Fischel JL, Formento P, Renee $\mathrm{N}$ and Milano G: A role for dihydropyrimidine dehydrogenase and thymidylate synthase in tumour sensitivity to fluorouracil. Eur J Cancer 30A: 1517-1522, 1994.

18. Thybusch-Bernhardt A, Beckmann S and Juhl H: Comparative analysis of the EGF-receptor family in pancreatic cancer: expression of HER-4 correlates with a favourable tumor stage. Int J Surg Investig 2: 393-400, 2001.

19. Drebin JA, Link VC, Stern DF, Weinberg RA and Greene MI: Down-modulation of an oncogene protein product and reversion of the transformed phenotype by monoclonal antibodies. Cell 41: 697-706, 1985.

20. Sarup JC, Johnson RM, King KL, Fendly BM, Lipari MT, Napier MA, Ullrich A and Shepard HM: Characterization of an anti-p185HER2 monoclonal antibody that stimulates receptor function and inhibits tumor cell growth. Growth Regul 1: 72-82, 1991.

21. Baselga $\mathrm{J}$ and Albanell $\mathrm{J}$ : Mechanism of action of anti-HER2 monoclonal antibodies. Ann Oncol 12 (suppl 1): 35-41, 2001.

22. Arteaga CL, Winnier AR, Poirier MC, Lopez-Larraza DM, Shawver LK, Hurd SD and Stewart SJ: p185c-erb-2 signaling enhances cisplatin-induced cytotoxicity in human breast carcinoma cells: association between an oncogenic receptor tyrosine kinase and drug-induced DNA repair. Cancer Res 54: 3758-3765, 1994.

23. Pietras RJ, Fendly BM, Chazin VR, Pegram MD, Howell SB and Slamon DJ: Antibody to HER-2/neu receptor blocks DNA repair after cisplatin in human breast and ovarian cancer cells. Oncogene 9: 1829-1838, 1994.

24. Baselga J, Norton L, Masui H, Kim YM and Mendelsohn J: Recombinant humanized anti-HER2 antibody (Herceptin) enhances the antitumor activity of paclitaxel and doxorubicin against HER2/neu overexpressing human breast cancer xenografts. Cancer Res 58: 2825-2831, 1998.

25. Pegram M, Hsu S, Lewis G, et al: Inhibitory effects of combinations of HER-2/neu antibody and chemotherapeutic agents used for treatment of human breast cancers. Oncogene 18: 2241-2251, 1999.

26. Fujimoto-Ouchi K, Sekiguchi F and Tanaka Y: Antitumor activity of combinations of anti-HER-2 antibody trastuzumab and oral fluoropyrimidines capecitabine/5'-dFUrd in human breast cancer models. Cancer Chemother Pharmacol 49: 211-216, 2002.

27. Baselga J, Norton L, Masui H, Pandiella A, Coplan K, Miller WH Jr and Mendelsohn J: Antitumor effects of doxorubicin in combination with anti-epidermal growth factor receptor monoclonal antibodies. J Natl Cancer Inst 85: 1327-1333, 1993. 